\title{
The Influence of Iterative Reconstruction Technique on the Diagnosis of Coronary Artery Calcification
}

\section{Ahmed Khamis AlShamakhi, MSc ${ }^{1 *}$, Rakesh Malhari Jamkhandikar, MD ${ }^{1}$, Yahya Mohammed AlWahshi, MD² and Sathiya Murthi Panchatcharam, MSc ${ }^{3}$}

${ }^{1}$ Department of Radiology and Imaging, Armed Forces Hospital, Oman

${ }^{2}$ Department of Medicine, Armed Forces Hospital, Oman

${ }^{3}$ Department of Studies and Research, Oman Medical Specialty Board, Oman

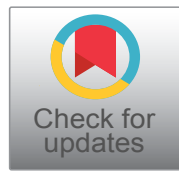

*Corresponding author: Ahmed Khamis AlShamakhi, MSc, Department of Radiology and Imaging, Armed Forces Hospital, Muscat, P.O Box 726, Post Code 111 Seeb, Oman, Tel: +968-24331757; +968-92470761, Fax: +968-24-331348

\begin{abstract}
Objectives: This study aimed to assess the impact of iterative reconstruction (IR) algorithms for the evaluation of coronary artery calcification (CAC) in terms of image quality and subjective diagnostic performance.
\end{abstract}

Methods: This study was performed in a single center and written informed consent was obtained from all patients. Thirty-one consecutive patients (26 men/5 women) underwent CT calcium score to rule out CAC. Image data were reconstructed with both; filtered back projection (FBP) and different levels of IR algorithms. Both the qualitative and quantitative image quality and subjective diagnostic performance were compared; Agatston scores and calcium density were measured for a total of 100 coronary arteries.

Results: Quantitatively; image noise was substantially reduced with high levels of IR reflecting high significances of noise level $(p<0.001)$. In terms of subjective diagnostic performance, it was observed with increased IR levels; $49 \%$ of coronary arteries showed decrease, $14 \%$ showed increase, $32 \%$ were constant and $5 \%$ of coronary arteries showed fluctuation in total Agatston scores. Certain Agatston scores were not detected in coronary arteries in some levels of IR algorithms.

Conclusions: IR resulted in substantial noise reduction and improved in both signal-to-noise ratio (SNR) and contrast-to-noise ratio (CNR). Higher levels of IR might lead to disappearance or underestimation of detectable calcium in coronary arteries with low calcium burden. Iterative reconstruction technique should be used with caution for better calcium quantification.

\author{
Keywords \\ Arteries, Calcium, Tomography
}

\section{Introduction}

Coronary artery disease (CAD) is the leading cause of morbidity and mortality in most developed countries throughout the world $[1,2]$. The continuing advances of multislice computed tomography (MSCT) have provided ongoing opportunities to improve the current practice in computed tomography (CT) clinical applications and in particular in cardiovascular imaging [3-6]. Quantification of coronary artery calcification (CAC) has been shown to be reliable, reproducible, and predictive of cardiovascular risk [7-14].

CAC is associated with arterial stiffness which ultimately increases risks for adverse cardiovascular events [15]. The extent of coronary artery calcification is strongly correlated with the degree of atherosclerotic disease and the rate of future cardiac events $[15,16]$.

$\mathrm{CT}$ is considered the modality of choice for assessment and quantification of coronary calcium score (CCS) [17]. CCS is considered as a well-known indicator of atherosclerotic plaque for which the prognostic value has been well established $[18,19]$. The Agatston score has been defined by Agatston and Janowitz and dates back into the 1980s [20]. This score still represents the most common established method for CCS quantification [21-23].

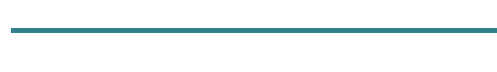

Citation: AlShamakhi AK, Jamkhandikar RM, AlWahshi YM, Panchatcharam SM (2021) The Influence of Iterative Reconstruction Technique on the Diagnosis of Coronary Artery Calcification. Int Arch Cardiovasc Dis 5:033. doi.org/10.23937/2643-3966/1710033 Accepted: January 21, 2021: Published: January 23, 2021

Copyright: (c) 2021 AlShamakhi AK, et al. This is an open-access article distributed under the terms of the Creative Commons Attribution License, which permits unrestricted use, distribution, and reproduction in any medium, provided the original author and source are credited.
} 
Table 1: Study population and different cardiovascular risk factors.

\begin{tabular}{|c|c|}
\hline Specifications & Total \\
\hline \multicolumn{2}{|l|}{ Population } \\
\hline Number of patients & 31 \\
\hline Age (years, mean \pm SD) & $57.1 \pm 9.2$ \\
\hline Gender (M/F) & $26 / 5$ \\
\hline BMI & $28.0 \pm 5.2$ \\
\hline Heart rate $(b p m ;$ mean $\pm S D)$ & $60.7 \pm 8.9$ \\
\hline \multicolumn{2}{|l|}{ Cardiovascular risk factors } \\
\hline Typical chest pain & 18 \\
\hline Known hypertension & 5 \\
\hline Coronary artery disease & 2 \\
\hline Ischemic heart diseases & 6 \\
\hline
\end{tabular}

BMI: Body Mass Index; BPM: Beat Per Minute; M/F: Male/ Female; SD: Standard Deviation

A high level of reproducibility for the measurement of coronary artery calcification is crucial; thus, effects of reconstruction algorithms on calcium scoring have been reported [24]. In comparison with standard filtered back projection (FBP), iterative reconstruction (IR) techniques result in substantial image noise reduction, and thus improve diagnostic image quality $[25,26]$. However; the current literature has contradictory on the effect of IR on the quantification of coronary calcification $[3,17,21,24,25,27,28]$. Therefore, this study aimed to define the threshold for the size and density in detection of CAC.

\section{Methods}

\section{Study population}

This study was performed in a single center, military base hospital, Oman; and approved by the Research and Ethical Committee Board. A written informed consent was obtained from all patients. Thirty-one consecutive adult patients were referred by cardiologist for CT coronary angiography (CCTA) for various clinical indications enrolled prospectively in this study (Table 1).

Exclusion criteria were renal insufficiency, pregnancy, asthma, patients with previous adverse reaction from iodinated contrast media and patients unable to consent.

All patients underwent a 64-MSCT scan examination of the calcium score as part of their CCTA (Brilliance; Philips Medical System, Best, Netherlands). The scan parameters were; axial scan mode; collimation, $40 \times$ 0.625 ; thickness, $2.5 \mathrm{~mm}$; increment, $2.5 \mathrm{~mm}$; rotation time, $0.4 \mathrm{~s}$; field of view, $220 \mathrm{~mm}$; matrix, $512 \times 512 ; \mathrm{kV}$, 120; mA, 60; filter, standard (B) and iDose4 iterative reconstruction technique (Philips Medical System, Best, Netherlands). Image data was reconstructed with an electrocardiogram gated window at $75.0 \%$ of the $R-R$ interval. Both qualitative and quantitative image analysis was performed in a dedicated CT diagnostic workstation

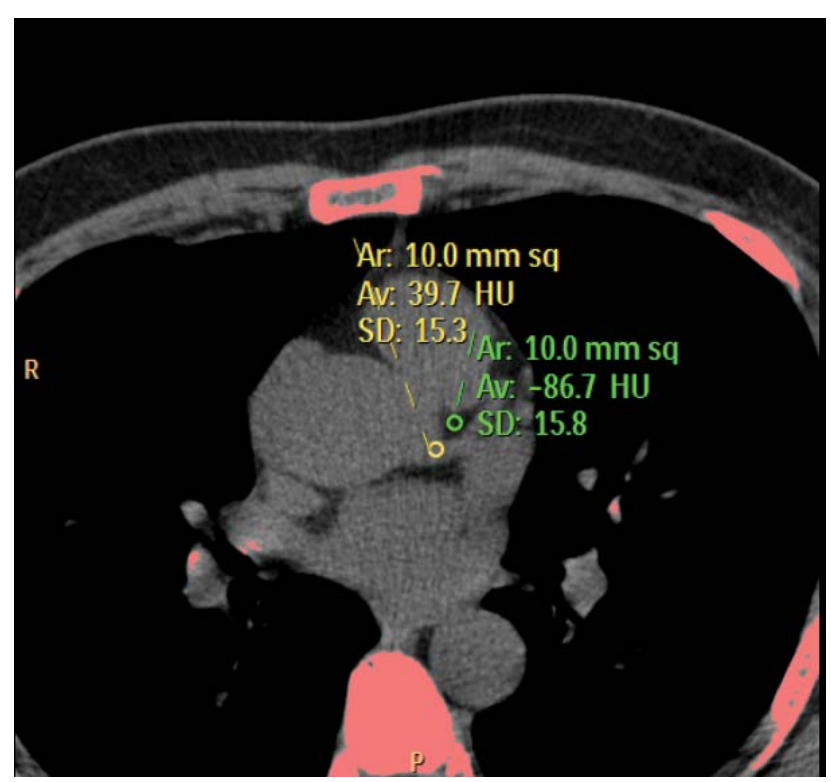

Figure 1: Illustrates the position of region of $\mathrm{ROI}$ in two different regions for calculating noise, SNR, and CNR. All ROls were drawn in a homogeneous region avoiding calcified areas.

CNR: Contrast-to-Noise ratio; ROI: Region of interest; SNR: Signal-to-noise ratio

(Extended Brilliance Workspace, version 4.5.5, Philips HealthCare).

\section{Image analysis}

Qualitative analysis: Qualitative analysis was performed by two readers; Radiologist and Cardiologist with extensive experience of 13 and 12 years, respectively, in cardiac CT image interpretation. Both readers were blinded to the reconstruction method (FBP and different levels of IR). Each reader was asked to select the most preferable reconstructed data from his subjective prospective in each scanned case.

Quantitative analysis: Quantitative analysis was determined by measuring noise, signal-to-noise ratio (SNR), and contrast-to-noise ratio (CNR) in the same cut of various data sets that reconstructed by FBP and different levels of IR algorithms.

As per Shen, et al. [8] CT densities were measured at aortic root cranial to left coronary ostium, left main coronary artery, and epicardial fat surrounding left main coronary artery. Image noise was defined as the standard deviation of CT density at aortic root; cranial to left coronary ostium. SNR was drawn by dividing the CT density of left main coronary artery by image noise. As for CNR, CT density of left main coronary artery was first subtracted by that of epicardial fat surrounding left main coronary artery and then divided by image noise (Figure 1).

\section{Subjective diagnostic performance}

Subjective diagnostic performance was analyzed in 100 coronary vessels in terms of total calcium score (Agatston) and the Hounsfield unit (HU) for each measured 
calcification in different reconstruction algorithms.

Statistical analysis: For statistical analysis all data was analyzed using MedCalc statistical software (version 18.2.1 MedCalc Software bvba, Ostend, Belgium, http://medcalc.org; 2018). Categorized variables were presented as number and percentages, continuous variables were presented with mean and standard deviation. Agreement was computed using weighted Kappa and comparisons were tested using Wilcoxon signed rank test. The two-sided $P$-values of less than 0.05 were considered to indicate a statistical significance.

\section{Results}

\section{Qualitative assessment of image quality}

The subjective assessment showed that there is poor inter-rater agreement between the two readers representing $29 \%$. Radiologist preference was image with high level of iterative ( $\geq$ IR4) and Cardiologist preference was image with low level of iterative $(\leq I R 3)$ representing $87 \%$ and $80 \%$, respectively.

\section{Quantitative assessment of image quality}

In iterative reconstructions; noise level showed a substantial reduction compared to FBP reflecting high significant change. The mean values were ranging between 18.04-10.33, which is highly significant ( $p<$ 0.001 ) (Table 2). In addition, both SNR and CNR showed an increasing trend with increasing strength of iterative reconstruction ranging from 2.05-3.60 and 8.24-14.47 respectively $(p<0.001)$.

\section{Subjective diagnostic performance}

Clinically coronary calcification in the 100 analyzed coronary vessels were seen in right coronary artery, left main, left anterior descending and circumflex branch of left coronary artery were 24, 3, 50 and 23, respectively.

It was observed with increased IR levels, the behavior of coronary arteries in terms of total Agatston scores and Hounsfield unit (HU) were not steady. By increasing the IR levels; the study showed decreases in total Agatston score in $49 \%$ of coronary arteries, no change in $32 \%$, increases in $14 \%$ and showed fluctuations in $5 \%$. In terms of $\mathrm{HU} ; 46 \%$ of coronary arteries decreased, $32 \%$ increased and $22 \%$ showed fluctuation in HU density.

These findings were expressed statistically reflecting very high differences. All the comparisons showed high

Table 2: Association between different level of iterative reconstruction with imaging parameters (Noise, SNR and CNR).

\begin{tabular}{|c|c|c|c|c|c|c|c|c|c|}
\hline \multirow{2}{*}{ Algorithm } & \multicolumn{2}{|c|}{ Noise } & \multirow[t]{2}{*}{ p-Value } & \multicolumn{2}{|c|}{ SNR } & \multirow[t]{2}{*}{ p-Value } & \multicolumn{2}{|c|}{ CNR } & \multirow[t]{2}{*}{ p-Value } \\
\hline & Mean & Sd & & Mean & Sd & & Mean & Sd & \\
\hline IR1 & 18.04 & 5.44 & \multirow[t]{6}{*}{$<0.001$} & 2.05 & 0.88 & \multirow[t]{6}{*}{$<0.001$} & 8.24 & 2.67 & \multirow[t]{6}{*}{$<0.001$} \\
\hline IR2 & 16.72 & 5.10 & & 2.20 & 0.95 & & 8.87 & 2.91 & \\
\hline IR3 & 15.37 & 4.98 & & 2.40 & 1.0 & & 9.68 & 3.23 & \\
\hline IR4 & 13.77 & 4.79 & & 2.69 & 1.19 & & 10.76 & 3.65 & \\
\hline IR5 & 12.17 & 4.58 & & 3.04 & 1.37 & & 12.26 & 4.39 & \\
\hline IR6 & 10.33 & 4.06 & & 3.60 & 1.69 & & 14.47 & 5.42 & \\
\hline
\end{tabular}

CNR: Contrast-to-Noise Ratio; SD: Standard Deviation; SNR: Signal-to-Noise Ratio

Table 3: Comparison of FBP TCS and HU in different levels of IR.

\begin{tabular}{|c|c|c|c|c|}
\hline \multicolumn{2}{|c|}{$\begin{array}{l}\text { Baseline } \\
\text { Mean (sd) }\end{array}$} & \multicolumn{2}{|c|}{ IR levels } & \multirow{2}{*}{$\begin{array}{r}\text { p-Value }^{*} \\
0.001\end{array}$} \\
\hline \multirow{6}{*}{ FBP-TCS } & \multirow{6}{*}{$6.66(10.59)$} & IR1 & $6.57(10.57)$ & \\
\hline & & IR2 & $6.54(10.56)$ & $<0.001$ \\
\hline & & IR3 & $6.51(10.51)$ & $<0.001$ \\
\hline & & IR4 & $6.46(10.46)$ & $<0.001$ \\
\hline & & IR5 & $6.41(10.43)$ & $<0.001$ \\
\hline & & IR6 & $6.37(10.42)$ & $<0.001$ \\
\hline \multirow{6}{*}{ FBP-HU } & \multirow{6}{*}{$183.29(46.34)$} & IR1 & $172.88(66.46)$ & 0.001 \\
\hline & & IR2 & $165.79(76.62)$ & $<0.001$ \\
\hline & & IR3 & $166.0(80.51)$ & 0.001 \\
\hline & & IR4 & $160.24(84.19)$ & 0.002 \\
\hline & & IR5 & $153.51(91.41)$ & 0.001 \\
\hline & & IR6 & $149.65(95.51)$ & 0.002 \\
\hline
\end{tabular}

"Wilcoxon Signed Ranks Test

FBP: Filtered Back Projection; HU: Hounsfield Unit; IR: Iterative Reconstruction; SD: Standard Deviation; TCS: Total Calcium Score 
statistical significances ( $P$-value 0.001 to $<0.001)$. In terms of HU density, it also showed higher differences in all levels ( $P$-value 0.002 to $<0.001)$ (Table 3).

The study also showed that certain Agatston scores were not detected in some coronary arteries in some levels of iterative ranging between 0.15-0.92 sqmm. Agatston score (Figure 2 and Figure 3 ) with various densities ranging between 130-159 HU.
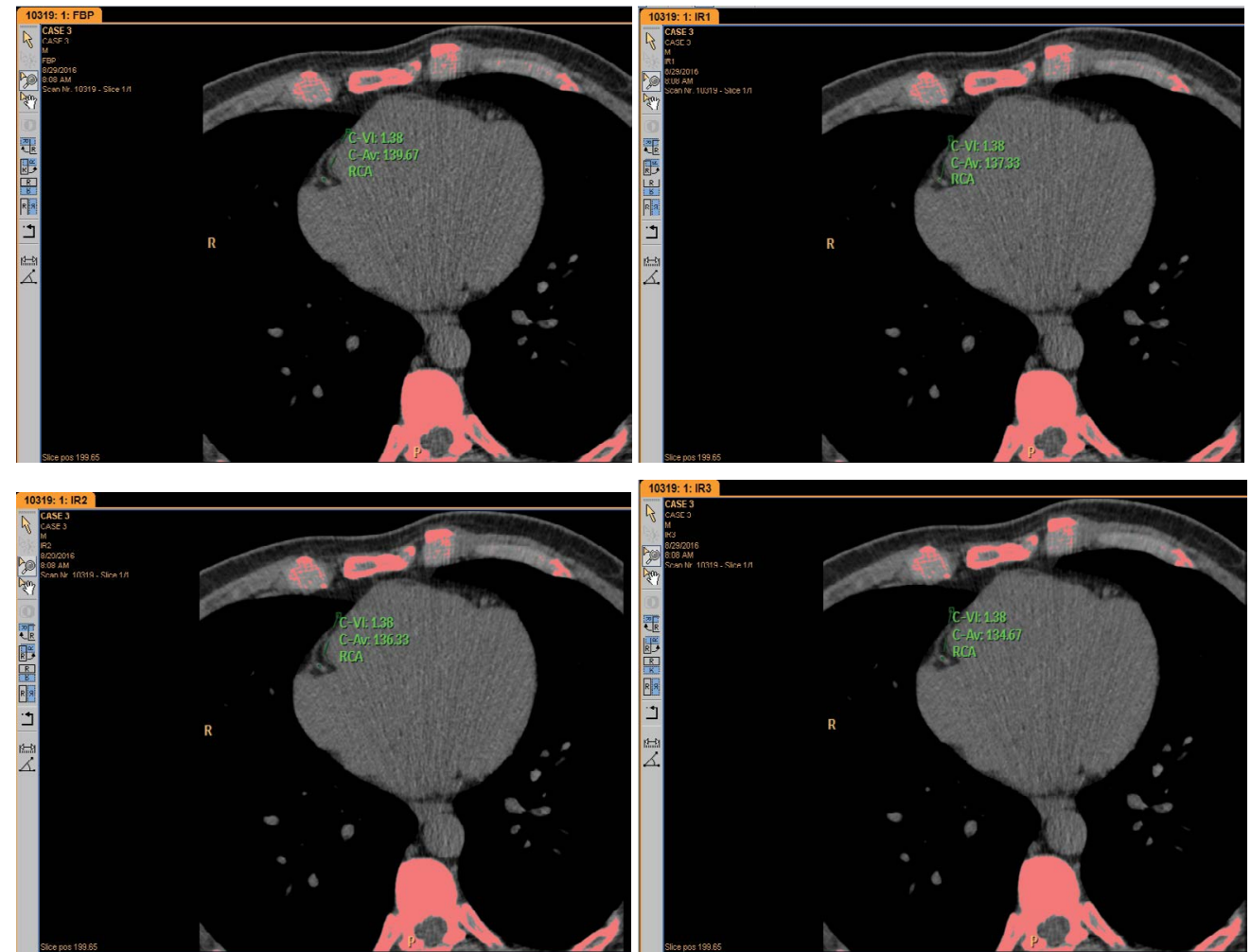

Figure 2: Agatston score is detected in FBP, IR 1, IR 2 \& IR 3 with variation in density level (HU). FBP: Filter Back Projection; HU: Hounsfield Unit; IR: Iterative Reconstruction
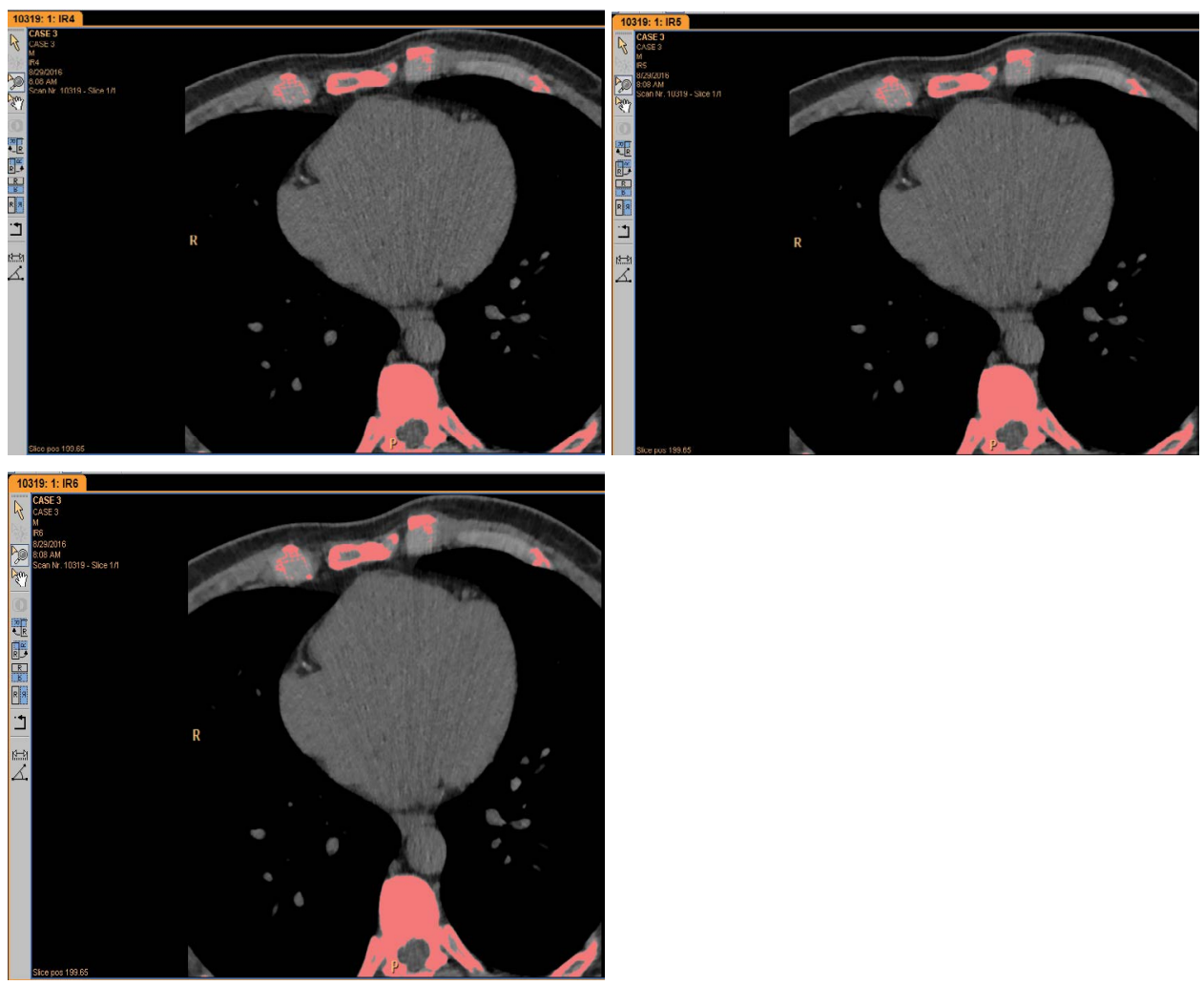

Figure 3: Agatston score is not detected in IR 4, IR 5 \& IR 6.

IR: Iterative Reconstruction 


\section{Discussion}

Coronary artery calcification (CAC) is highly prevalent in patients with coronary artery disease (CAD) and is associated with major adverse cardiovascular events. To date, there is no established treatment for CAC, however, a reliable tool is needed to determine the actual presence and extent of calcified coronary artery plaque [15]. Calcium scoring has been shown to be a reliable and non-invasive technique to independently predict cardiovascular events as well improve traditional risk prediction models. CAC is also considered as a subclinical marker of atherosclerosis for which the prognostic value has been well established [14].

Calcified atherosclerotic plaques are not uniformly distributed throughout the coronary artery tree. In the 100 analyzed coronary arteries; $50 \%$ of coronary calcifications were in the left anterior descending (LAD), 24\% were seen in right coronary artery (RCA), $23 \%$ in the circumflex branch of left coronary artery and $3 \%$ of coronary calcifications in the left main. Lesions were predominantly located in the LAD and this finding is consistent with Wasilewski, et al. [16] who stated that the possible explanation for this phenomenon could be the dynamic compression of the tunneled septal branches, which may result in distributed blood flow in the adjacent LAD segment (milking effect).

Subjective assessment has reflected very poor inter-rater agreement between the two readers. This can be explained by the fact that each reviewer according to his specialty, concentrates on different areas of interest. For example, the Radiologist apart from reviewing coronary arteries, also assesses other anatomical parts such as lungs, bones and soft tissues; whereas Cardiologist tends to focus mainly on coronary arteries.

Quantitative image quality analysis in term of image noise was reported to be significantly lower with IR algorithm compared to FBP. Also, both SNR and CNR showed significant improvement with increasing the levels of iterative reconstruction. This phenomenon is consistent to AlShammakhi \& Sun [29] in their retrospective study and many others researchers $[8,11,24,25]$.

In $49 \%$ of coronary arteries, there was reduction in total Agatston scores with higher strength levels of IR. This phenomenon was reported in many studies $[3,17,21,24]$; and most likely caused as reported by Gassenmaier, et al. and AlShammakhi \& Sun [21,29] as a result of sharpening the calcifications and thus reducing the number of pixels with densities over the scoring threshold and thus suppressing the blooming artifacts and improve estimation of the true degree of luminal narrowing.

By increasing the IR levels; the study showed decreases in total CCS in $49 \%$ of total coronary arteries, no change in $32 \%$, increases in $14 \%$ and showed fluctuation in CCS in $5 \%$ of the coronary arteries. This phenomenon was irrelevant to body mass index (BMI) and patient gender; and only can be explained due to the adjacent anatomical structures to that particular calcification or the presence of slight artifact due to breathing. Subsequent studies assessing the behavior of coronary calcification based to location, surrounding structures and possibility of breathing artifacts would be required.

Clinically, a balance of IR strength has to be found to avoid higher or lower estimate of the Agatston scores, therefore; this study is not recommending higher level of IR in coronary calcium score assessment and should not exceed more than level 2 . The study proved that higher level leads to disappearance of detectable calcium particularly in small scores ranging between 0.15 to 0.92 sqmm. Agatston scores.

This study has two main limitations that should be taken into consideration; first, sample size was relatively small. However; the study results were able to reflect the differences and draw a conclusion subjectively and objectively. Second, results were drawn from a single MSCT and the effect of iterative from other vendors might be different.

\section{Conclusion}

Knowledge regarding the presence or absence of CAC can lead to a better estimation of risk which the potential management relies on. Iterative reconstruction algorithm improves the diagnostic image quality substantially, however, higher IR level might lead to disappearance or underestimate of detectable calcium in coronary arteries with low calcium burden.

Our study proved that with higher IR (IR 3 and above) the Agatston calcium score upto $0.9 \mathrm{sqmm}$. and density of $138 \mathrm{HU}$ can be missed which in turn can lead to underestimation of total Agatston score. Hence, IR technique should be used with caution for better calcium quantification and iterative reconstruction algorithm above IR2 is not recommendable.

\section{Conflict of Interest}

The authors declare no conflict of interest.

\section{References}

1. Divakaran S, Cheezum M, Hulten E, Bittencourt M, Silverman M, et al. (2014) Use of cardiac CT and calcium scoring for detecting coronary plaque: Implications on prognosis and patient management. Br J Radiol 88: 20140594.

2. Bijl N, Joemai R, Geleijns J, Bax J, Schuijf J, et al. (2010) Assessment of Agatston coronary artery calcium score using contrast-enhanced CT coronary angiography. AJR 195: 1299-1305.

3. Kurata A, Dharampal A, Dedic A, Feyter P, Krestin G, et al. (2013) Impact of iterative reconstruction on CT coronary calcium quantification. Eur Radiol 23: 3246-3252.

4. Valzen J, Schuijf J, Graaf F, Boersma E, Pundziute G, et al. (2011) Diagnostic performance of non-invasive multidetector computed tomography coronary angiography to detect 
coronary artery disease using different endpoint: Detection of significant stenosis vs. detection of atherosclerosis. Eur Heart J 32: 637-645.

5. AlShammakhi A, Sun Z (2015) A systematic review of image quality, diagnostic value and radiation dose of coronary CT angiography using iterative reconstruction compared to filtered back projection in the diagnosis of coronary artery disease. J Med Imaging Health Inf 5: 96-102.

6. Hou Z, Lu B, Gao Y, Jiang S, Wang Y, et al. (2012) Prognostic value of coronary CT angiography and calcium score for major adverse cardiac events in outpatients. JACC Cardiovasc Imaging 5: 990-999.

7. Ferencik M, Mayrhofer T, Puchner S, Lu M, Maurovich-Horvat $P$, et al. (2015) Computed tomography-based high-risk coronary plaque score to predict acute coronary syndrome among patients with acute chest pain-Results from ROMICAT II trail. J Cardiovasc Comput Tomogr 9: 538-545.

8. Shen H, Dai G, Luo M, Duan C, Cal W, et al. (2015) Image quality and radiation dose of $\mathrm{CT}$ coronary angiography with automatic tube current modulation and strong adaptive iterative dose reduction three-dimensional (AIDR3D). PLoS One 10: e0142185.

9. Yin W, Lu B, Hou Z, Li N, Han L, et al. (2013) Detection of coronary artery stenosis with sub-milliSievert radiation dose by prospective ECG-triggered high-pitch spiral CT angiography and iterative reconstruction. Eur Radiol 23: 2927-2933.

10. Feng R, Tong J, Liu X, Zhao Y, Zhang L (2017) High-pitch coronary CT angiography at $70 \mathrm{kVp}$ adopting a protocol of low injection speed and low volume of contrast medium. Korean J Radiol 18: 763-772.

11. Arcadi T, Maffer E, Mantini C, Guaricci A, Grutta L, et al. (2015) Coronary CT angiography using iterative reconstruction vs. filtered back projection: Evaluation of image quality. Acta Biomed 86: 77-85.

12. Zhang L, Qi L, Cecco C, Zhou C, Spearman J, et al. (2014) High-pitch coronary CT angiography at $70 \mathrm{kVp}$ with low contrast medium volume. Comparison of 80 and $100 \mathrm{kVp}$ high-pitch protocols. Medicine (Baltimore) 93: e92.

13. Chinnaiyan K, Bilolikar A, Walsh E, Wood D, DePetris A, et al. (2014) CT dose reduction using prospectively triggered or fast-pitch spiral technique employed in cardiothoracic imaging (the CT dose study). J Cardiovasc ComputTomogr 8: 205-214.

14. Blaha M, Mortensen M, Kianoush S, Tota-Maharaj $R$, Cainzos-Achirica M (2017) Coronary artery calcium scoring, Is it time for a change in methodology? JACC Cardiovasc Imaging 10: 923-937.

15. Liu W, Zhang Y, Yu C, Ji Q, Cai M, et al. (2015) Current understanding of coronary artery calcification. J GeriatrCardiol 12: 668-675.

16. Wasilewski J, Niedziela J, Osadmik T, Duszanska A, Sraga W, et al. (2015) Predominant location of coronary artery atherosclerosis in the left anterior descending artery. The impact of septal perforators and the myocardial bridging effect. Imaging Technique 12: 379-385.

17. Werf N, Willemink M, Greuter M, Leiner T (2017) Influence of dose reduction and iterative reconstruction on CT calcium scores: A multi-manufacturer dynamic phantom study. Int J Cardiovasc Imaging 33: 899-914.

18. Choi A, Leifer E, Yu J, Shanbhag S, Bronson K (2016) Prospective evaluation of the influence of iterative reconstruction on the reproducibility of coronary calcium quantification in reduced radiation dose 320 detector row CT. J Cardiovasc Comput Tomogr 10: 359-363.

19. Pressman G, Crudu V, Parameswaran-Chandrika A, Romero-Corral A, Purushottam B, et al. (2011) Can total cardiac calcium predict the coronary calcium score? Int J Cardiol 146: 202-206.

20. Leherbauer L, Sonneck-Koenne C, Zakavi S, Haoula D, Heydari B, et al. (2011) Coronary artery calcium (CAC) score-a prognostic tool in coronary artery disease? Iran J Nucl Med 19: 46-51.

21. Gassenmaier T, Allmendinger T, Kunz A, Veyhl-Wichmann M, Ergun S, et al. (2017) In vitro evaluation of a new iterative reconstruction algorithm for dose reduction in coronary artery calcium scoring. Acta Radiol Open 6: 2058460117710682.

22. Sandfort V, Bluemke D (2017) CT calcium scoring. History, current status and outlook. Diagn Interv Imaging 98: 3-10.

23. Shabestari A (2013) Coronary artery calcium score: A review. Iran Red Cres Med J 15: e16616.

24. Gebhard C, Fiechter M, Fuchs T, Ghadri J, Herzog B, et al. (2013) Coronary artery calcium scoring: Influence of adaptive statistical iterative reconstruction using 64-MSCT. Int J Cardiol 167: 2932-2937.

25. Renker M, Nance J, Schoepf U, O'Brien T, Zwemer P, et al. (2011) Evaluation of heavily calcified vessels with coronary CT angiography: Comparison of iterative and filtered back projection image reconstruction. Radiology 260: 390-399.

26. Greffier J, Macri F, Larbi A, Fernandez A, Khasanova E, et al. (2015) Dose reduction with iterative reconstruction:Optimization of CT protocols in clinical practice. Diagn Interv Imaging 96: 477-486.

27. Schindler A, Vliegenthart R, Schoepf U, Blanke P, Ebersberger $U$, et al. (2014) Iterative image reconstruction techniques for CT coronary artery calcium quantification: Comparison with traditional filtered back projection in vitro and vivo. Radiology 270: 387-393.

28. Williams M, Weir N, Uren N, McKillop G, Newby D, et al. (2012) The effect of iterative reconstruction on computed tomography coronary artery calcium score. ESR.

29. AIShammakhi A, Sun Z (2015) Coronary CT angiography with use of iterative reconstruction algorithm in coronary stenting: A systemic review of image quality, diagnostic value and radiation dose. J Med Imaging Health Inf 5: 103109. 